\section{LNCRNA MALAT1 REGULATED SERPINB6B SIGNALING SUPPRESSES PYROPTOSIS AND GOVERNS BREAST CANCER DORMANCY REACTIVATION AND THROUGH IMMUNE EVASION}

Dhiraj Kumar*, Filippo Giancotti. UT MD Anderson Cancer Center, Houston, TX, USA

Background Metastatic relapse is the major causes of mortality in patients with cancer and occur due to metastatic reactivation of dormant tumor cells. Early dissemination of tumor cells undergoing a protected period of dormancy in the target organs potentially explains this prevalent clinical behavior. ${ }^{1-4}$ Long non-coding RNAs (lncRNAs) are involved in various biological processes and diseases. Malat1 is one of the most abundant and highly conserved nuclear lncRNAs and have shown the associated with metastasis and serving as a predictive marker for various tumor progression. ${ }^{5}$ However, the correlation of tumor intrinsic lncRNAs in regulation of tumor dormancy and immune evasion is largely unknown.

Methods Using an in vivo screening platform for the isolation of genetic entities involved in either dormancy or reactivation of breast cancer tumor cells, we have identified Malat1 as a positive mediator of metastatic reactivation. ${ }^{4}$ To dissect the functional role of Malat1 in metastatic reactivation, we developed a clean Malat1 knockout (KO) model using paired gRNA CRISPR-Cas9 in metastatic murine syngeneic breast cancer. As proof of concept we also used inducible knockdown system under in vivo models. To delineate the immune microenvironment, we used single cell RNA-seq, ChIRP-seq, multicolor flowcytometry, RNA-FISH, and coculture experiments.

Results Our data revealed that deletion of Malat1 induces dormancy and attenuated the metastatic colonization resulting in long-term survival of syngeneic mice model. In contrast, overexpression of Malat1 leads to metastatic reactivation of dormant breast cancer cells. Interestingly, 4T1-Malat1 KO dormant breast cancer cells exhibit metastatic outgrowth in $\mathrm{T}$ cells defective mice. Our single-cell RNA-seq and multicolor flowcytometry evaluation reveal enhanced $\mathrm{T}$ cells and reduced neutrophils proportions in mice with Malat1 KO cells. This indicates a critical role of immune microenvironment via Malat1-dependent immune evasion. Additionally, Malat1 KO inhibits cancer stemness properties. Similarly, RNA-seq and ChIRP-seq data suggest that $\mathrm{KO}$ of Malat1 hampers immune evasion and downregulates metastasis associated genes including Serpins and Wnts. Additionally, our data strongly suggests that Malat1 KO cells persists as non-proliferative dormant cells in lung due to $\mathrm{CD} 8+\mathrm{T}$ cell-umpired immune activity. Interestingly, rescue experiments suggest that Malat1 or Serpinb6b protects $\mathrm{T}$ cell-induced cell death and induces dormancy re-awakening thereby rescue the metastatic potential of 4T1 Malat1 KO cells. Combination of Malat1 ASO with double immune checkpoint inhibitors greatly affects the metastatic outgrowth in breast cancer.

Conclusions Taken together, our studies demonstrate that tumor intrinsic Malat1 regulates Serpinb6b that eventually controls immune evasion and promote dormancy metastatic reactivation.

Acknowledgements NGS data generated was supported by Core grant CA016672(ATGC) and NIH 1S10OD024977-01 award to the ATGC. Single cell RNA sequencing data was supported by the CPRIT Single Core grant RP180684. The Advanced Cytometry \& Sorting Core Facility is supported by NCI P30CA016672.

\section{REFERENCES}

1. Arun $G$, Diermeier $S$, Akerman $M$, et al. Differentiation of mammary tumors and reduction in metastasis upon Malat1 IncRNA loss. Genes Dev 2016 January 1;30 (1):34-51.

2. Filippo G Giancotti. Mechanisms governing metastatic dormancy and reactivation. Cell 2013 November 7;155(4):750-764.

3. Gao H, Chakraborty G, Lee-Lim AP, et al. The BMP inhibitor Coco reactivates breast cancer cells at lung metastatic sites. Cell 2012b;150:764-779.

4. Gao H, Chakraborty G, Lee-Lim AP, et al. Forward genetic screens in mice uncover mediators and suppressors of metastatic reactivation. Proc Natl Acad Sci U S A 2014 November 18;111(46):16532-16537.

5. Huang D, Chen J, Yang L, et al. NKILA IncRNA promotes tumor immune evasion by sensitizing $T$ cells to activation-induced cell death. Nat Immunol 2018;19:1112-1125.

http://dx.doi.org/10.1136/jitc-2021-SITC2021.931 